\title{
Introduction: synchrotron radiation time resolved concurrent experiments-a new Italian route to China
}

\author{
Plinio Innocenzi $\cdot$ Augusto Marcelli $\cdot$ Ziyu Wu
}

Published online: 1 November 2011

(C) Springer-Verlag 2011

On November 11, 2010 in Shanghai qualified Chinese and Italian scientists met for the first Bilateral Italo/Chinese Workshop on Synchrotron Radiation Time Resolved Concurrent Experiments: Advantages and Future Applications. The workshop had been conceived to increase the scientific exchange between Italy and the P.R. China with the main goal to promote the development of new and unique methods of investigation by the means of synchrotron radiation.

From its discovery in 1947 the development of synchrotron radiation sources and the growth of the related applications have been impressive and continuous all over the world, in particular in the major industrialized countries. In more than 50 years since its discovery, facilities around the world constantly evolved to provide beams in different forms, at different energies and with different properties for an incredible number of applications. Experimental researches based on this technology involve many academic disciplines and synchrotron radiation is nowadays an essential tool in many frontier disciplines. Because of its outstanding features, synchrotron radiation is a high-level multidisciplinary experimental platform that plays an essential role in many researches and technological applications, being universally acknowledged by the scientific community, the society as well as the governments. Actually, with more than 50 operational storage rings existing all over the world, laboratories based on synchrotron radiation sources are the most widely operated large-scale facilities with a total investment in the range of billions of dollars.

Synchrotron radiation helped to push forward a huge number of researches that led to important discoveries. The reconstruction of the atomic structure of many proteins or of the local structure of high critical temperature superconductors may give just an idea of the

P. Innocenzi $(\bowtie)$

Science and Technology Councellor, Embassy of Italy, San Li Tun, Dong Er Jie N.2,

Beijing 100600, People's Republic of China

e-mail: plinio.innocenzi@esteri.it

A. Marcelli

INFN, Laboratori Nazionali di Frascati, Via E. Fermi 40, 00044 Frascati, Rome, Italy

Z. Wu

National Synchrotron Radiation Laboratory, University of Science and Technology of China, 42\# HeZuoHua South Road, Hefei 230029, Anhui, People's Republic of China 
role of synchrotron radiation nowadays. Users have mainly driven the continuous growth of these researches, but applications are still expanding. The most recent ideas and ongoing developments provide benefits hardly imaginable just a few years ago. However, a new scenario emerges in this area that promises to be even more exciting. Thanks to the brilliance of the last generation of these sources, it is now possible to perform timeresolved experiments with a resolution previously unattainable; consequently, it appears now possible reconstructing the dynamics of many physical and chemical processes such as phase transitions and self-assembly processes. Order/disorder phenomena, nano-systems, low dimensional systems, surface and interface processes, even living systems can be now investigated on a very short timescale. However, for such challenging researches new methods have to be developed and implemented.

The bilateral workshop was a great opportunity to share knowledge and to exchange ideas between Italian scientists who had reached expertise on synchrotron radiation physics and technology and the very large Chinese scientific community operating in these facilities. According to the Chinese economic and scientific development plan and its foreseen long-term development trend, the role of large-scale radiation facilities will certainly grow in the next decade. In concomitance with the 40th anniversary of diplomatic relations between Italy and China, the workshop was conceived and actually has been also a great opportunity to discuss and plan the foreseen bilateral cooperation and initiatives in the field of synchrotron radiation. Among the many ideas, the possibility of establishing a dedicated Italian laboratory in China where the continuity of these highly fruitful bilateral experiences could be consolidated was also discussed.

Acknowledgments The organization of the workshop was made possible by the joint effort of several Chinese and Italian institutions: the Institute of High Energy Physics (IHEP), the Istituto Nazionale di Fisica Nucleare (INFN), the National Synchrotron Radiation Laboratory (NSRL) and the Shanghai Synchrotron Radiation Facility (SSRF).We gratefully acknowledge the personnel of the Scientific Office of the Italian Embassy in the P. R. China for the help during the workshop and the Redazione of Accademia Nazionale dei Lincei for co-operation in the preparation of this Supplement. 